\title{
ABOUT THE NATURE OF METEOR FLARES
}

\author{
V.A.Smirnov \\ National Academy of Communications, Odessa, Ukraine \\ smirnovw@tm.odessa.ua
}

\begin{abstract}
Whereas at development of the meteoric phenomenon there are processes in different temporary scales (more slow processes heat conductivity, mechanical rotation, destruction) and considerably faster (fluctuations of brightness of radiating plasma is like gas-dynamical laser), the observable flares of meteors differ in nature, and in appearance caused by character of radiation.

Continuing development of the meteoric phenomena in the Earth's atmosphere has been described by Astapovich I.S. (Astapovich I.S. 1958). The classification of meteor flashes which depends on the physical nature of the phenomenon is given below and the formulas of a physical theory representing these processes while moving the meteor are presented here.
\end{abstract}

Key words: Flares, Meteor stream, Gas-dynamical laser, Ambipolar diffusion, Kinetics of meteor plasma.

\section{Introduction}

Analyzing the flares of fast meteors of Perseid, Orionid, Leonid meteoric streams and similar to those flares with identical radiation character are allocated in meteors of the same streams. Some flares, like for example double flares at the end of meteor path, are difficult to explain with regular methods of meteoroid's mechanical destruction, as mechanical changes proceed incomparably more slowly than the processes of changing of observable meteoric plasma radiation (Smirnov, 1994, 2007).

The flares of slow bolides, rather slow massive meteors flying with speed less than $60 \mathrm{~km} / \mathrm{s}$ form radiation simultaneously in all so-called "spectral lines" of the meteor spectral image - "lines" are monochromatic images of a meteor and cannot be used for constructing growth curves or self-absorption calculation. - V.S. - . These meteor flares can be recurring with the frequency of a meteor body rotation while dumping the fused layers of a meteor body and other similar mechanical phenomena.

Fast alternation of flares in this case characterizes discarding of a meteor separate boiling luminous layers in process of penetration of a meteoric body deep into atmosphere with the appropriate boiling point of a substance (for iron $2735^{\circ} \mathrm{C}$ ). The "mechanical" flare spectral lines light up at once simultaneously for the whole spectrum.

During the penetration of a meteoroid into the more dense atmospheric layers, formed meteor plasma interacts both with the meteor body and environment continuously increasing in density atmospheric gas.

In the above shower meteors the shock waves formed during the meteor body flight, along with the ambipolar diffusion, considerably accelerate and strengthen thermal ionization and thereby formation of the meteor plasma, plasma "clot".

At over $60 \mathrm{~km} / \mathrm{s}$ speed of the shower meteors, meteor plasma moves along with meteoroid with over $60 \mathrm{~km} / \mathrm{s}$ speed and causes immediate radiation intensity transformations (Smirnov, 2000, 2003).

Penetration of the meteor into the denser atmospheric layers entails emerging plasma that may often appear as a plasma formation with radiation similar to that of a gasdynamical laser. The flares with almost complete loss of the meteor mass by evaporation appear during the inevitable dissipation of the meteor plasma into void. The process is identical to a gas-dynamical laser radiation.

The first to light up in the "lines", according to the Wien law, is the higher wavelength spectrum part followed by the whole set of spectral "lines". These "laser" flares were called type A.

In this shown way experiments with the observed gasdynamical laser luminescence can be done. The experiments should simulate expansion of plasma into void as a function of the time-related medium density enhancement that corresponds to air density from zero to a $60-70 \mathrm{~km}$ height atmosphere density. In this case attention should be paid to presence or absence of the flares similar to meteor ones.

In work (Shinsuke Abe at al., 2007) on Fig 1 is shown spectrum of Leonid fireball, in which imposing both types of flares most likely is shown (see Fig.1).

\section{Ambipolar diffusion}

For understanding the plasmoid formation mechanism while the fast meteor's movement belongs as a rule to the meteor shower, we should recall the formulas defining the phenomenon of the ambipolar diffusion.

Flying meteors are known to be surrounded with ionized air columns. Thus ambipolar diffusion occurs continuously with the resulting plasma-like meteor clot. Ambipolar diffusion is the primary process in relation to excitation and radiation of the meteor ionized trace.

During diffusion the stream of particles $\mathrm{j}$ is proportional to a gradient of concentration: 


$$
\mathrm{j}=-\mathrm{D} \nabla N
$$

where $\mathrm{D}$ is diffusion coefficient proportional to a $\mathrm{V}$ particle characteristic velocity and the length of free path $\lambda$. According to the Einstein ratio the diffusivity is proportional to mobility of $b$ particles and gas temperature $\mathrm{T}$ :

$$
\mathrm{D}=\mathrm{bT} / \mathrm{e} \text {, }
$$

where $\mathrm{e}$ is the elementary charge.

During diffusion of charged particles, the streams of electrons and ions are composed both of diffusional and directional ones in electrical field:

$$
\begin{aligned}
& j_{e}=-D_{e} \nabla N_{e}-b_{e} N_{e} E, \\
& j_{j}=-D_{j} \nabla N_{i}+b_{i} N_{i} E,
\end{aligned}
$$

where $\mathrm{E}$ is the intensity of the field resulting from separation of charges; $b_{e}, b_{i}$ are the mobilities of electrons and ions; $D_{e}, D_{i}$ are diffusivities of plasma electrons and ions; $\mathrm{N}_{\mathrm{e}}, \mathrm{N}_{\mathrm{i}}$ are concentrations of electrons and positive ions.

Let's mark mass of an electron $m_{e}$ and mass of positive heavy ion, $\mathrm{m}_{\mathrm{i}}$. From the ratio

$$
\frac{D_{e}}{D_{i}} \approx \frac{V_{e}}{V_{i}} \cong \sqrt{\frac{m_{i}}{m_{e}}}
$$

it is seen that the drift velocity of an electron is $2-3$ orders higher than the velocity of positive ions. If an ionized "electronic" trace formation time is $10^{-3} \mathrm{~s}$, then the time of filling this cylindrical trace with positive ions will be no less than $0.4 \mathrm{~s}$. The same mechanism is involved in plasma clot formation as well.

\section{Scattering kinetics of meteoric plasma}

Thus, considering the meteoric phenomenon development in real-time mode we can guess that by the end of the meteoroid particles evaporation and sublimation process the originated as a meteoric coma plasma clot loses its source of radiating particles replenishment.

As reference data suggest, at $90 \mathrm{~km}$ height an average length of air particles free run is $2.4 \mathrm{~cm}$, at $80 \mathrm{~km}$ height $0.4 \mathrm{~cm}$. So geometric meteor measures correspond to the ones of radiating meteoroid, and only these physical conditions give start to the process of heating and subsequent intensive luminescence of the meteoroid (Astapovich, 1958).

Heat conductivity according to the researched soil data at densit $\rho=0.5 \mathrm{~g} / \mathrm{cm}^{3}$ and $90 \%$ porosity is $0.48 \cdot 10^{-4}$ Joule/mm.s.K (Chudnovsky, 1976). The slow process of heat conductivity is responsible for meteoric body inertness preservation during flight and accounts for physical distinction between flares, such as types A and B.

During the meteor body radiating particles source disappearance the overall brightness of the meteor declines to full disappearance of solid mass. As was shown (Smirnov, 1994, 2007), a visible spectrum radiation is possible when radiating plasma velocity is decelerated down to $2-3 \mathrm{~km} / \mathrm{s}$ is. If the velocity of meteor plasma would actually stay equal to the Leonids, Orionids, or Perseids streams meteor velocity, the maximum in the spectrum energy distribution would be displaced into Xray part of the spectrum, which fact was never observed.

The extension of the terminal plasma clot into void can be described according to the law:

$$
\rho=\frac{m}{\frac{4}{3} \pi R^{3}}=\rho_{0}\left(\frac{t_{0}}{t}\right)^{3},
$$

Hence, the expression for a time scale is determined as

$$
t_{0}=\left(\frac{m}{\rho_{0} \frac{4}{3} \pi V^{3}}\right)^{1 / 3}
$$

In (5) and (6) $\rho_{0}$ is the initial density of a plasma clot and $m$ is mass of a meteoroid; the radius of a full sphere formed by plasma with density $\rho$ is determined according to formula: $\mathrm{R}=\mathrm{Vt}$. So: during the about $0,3 \mathrm{~s}$ average time the scattering of plasma occurs, it filling the sphere with $\mathrm{R}$ radius; attenuation of the meteoroid's brightness preceding a final bright flare occurs.

The kinetics equation for extending plasma (Smirnov, 1994, 2000, 2002; Biberman et al., 1982) related to ionization level

$$
x=\frac{N_{e}}{N},
$$

where $\mathrm{N}_{e}$ is concentration of electrons, and $\mathrm{N}$ is concentration of colliding particles, looks like:

$$
\frac{d x}{d t}=-\alpha N^{2} x^{3},
$$

where $\alpha$ is a recombination coefficient, $\alpha=A T_{e}^{-9 / 2}$.

In the above expression $\mathrm{A}$ is constant, $\mathrm{T}_{\mathrm{e}}$ - electronic temperature.

If degree of ionization is $\mathrm{x}=\mathrm{x}_{0}$ at $\mathrm{t}_{0}$, then the equation solution can be represented as:

$$
x=x_{0}\left[1+2 A x_{0}^{2} \int_{t_{0}}^{t} \frac{N^{2}(t) d t}{T_{e}^{3 / 2}(t)}\right]^{-1 / 2}
$$

Thus, as is seen from (8), the number of particles $\mathrm{N}$ under radiation source impact will increase within one second, with constant volume preserved. Actually while scattering, however, the volume increases proportionally to $\mathrm{R}^{3}$, and electronic temperature, as well as concentration of particles accordingly decrease. Numerical examples are cited in (Smirnov, 1994). 


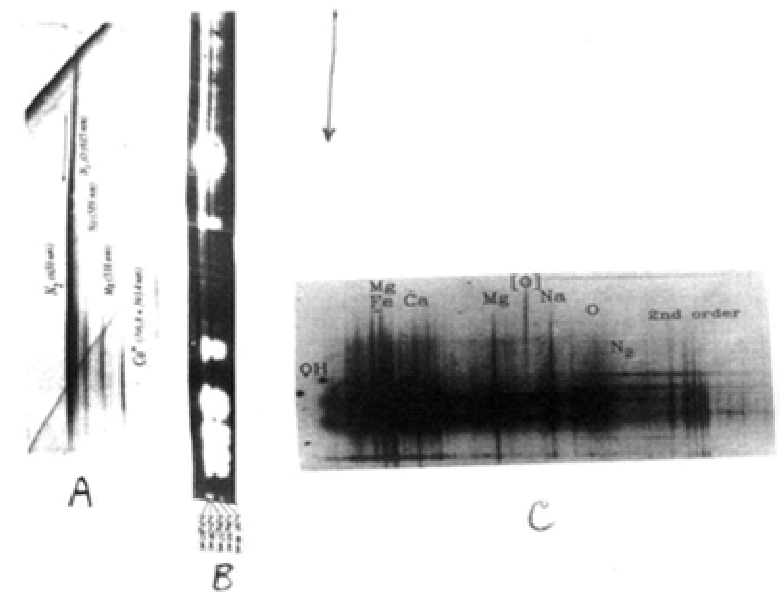

Figure 1:

A - type flare spectrum of Leonids, November 17/18, 1965 , photographed by the author. Final flare shows the «laser» radiation behavior.

B - the fireball spectrum with flares caused by rotation of a meteoric body moving in atmosphere, «mechanical» radiation behavior.

C - a Leonids spectrum from Japan (Abe et al., 2007). Radiation behavior suggests superimposed flares of $\mathrm{A}$ and B types.

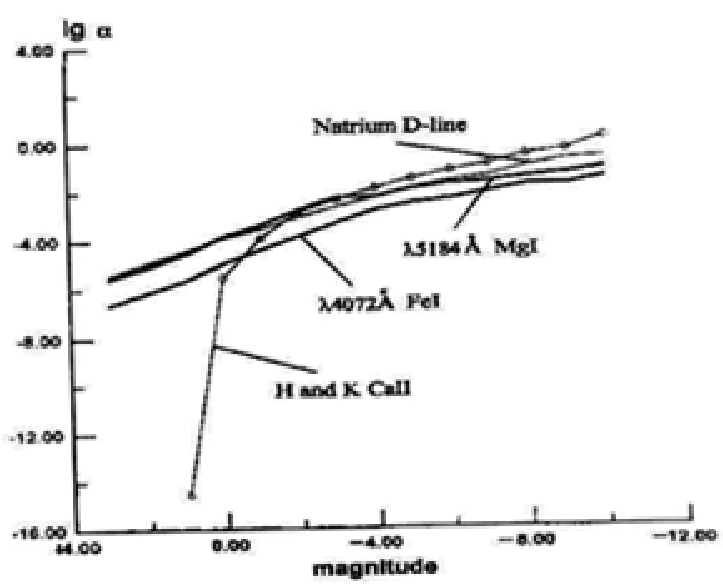

Figure 2: Coefficient of radiation intensification (on logarithmic scale) related to the meteor's stellar magnitude.

4. Conclusion: the mechanism of the Perseid', Orionid', Leonid' streams meteor characteristic flare

At an early stage of the meteor plasma clot scattering all relaxation processes are fast, and gas can be suggested to preserve a thermodynamic equilibrium.

Without the meteoroid's radiating mass replenishment which fact can be basically verified experimentally, in meteor plasma expanding through ambipolar diffusion the degree of particles excitation will increase through a shock wave due to recombination stream from above.

Populated in plasma particles are the levels nearing the edge of the continuous spectrum. A specific inversion is formed, followed by the maximum induced radiation.
With meteoric plasma disintegration, the excitation degrees of other atomic power levels should also increase until downward stream from every level due to spontaneous transitions and deactivation becomes predominating over a stream from continuous spectrum which will eventually decrease with particles concentration decrease.

The significant enough factors of radiation intensification due to such "macroquantum" effect are found only for fast stream meteors. As the meteor plasma is a mixture of various elements, hence excitation of the basic element brings forth intensification of others, as the experiments show (Smirnov, 1994).

If we admit that radiation from a final flare is produced by particles resulting from the inversed population of the particle levels, we can calculate coefficient of possible luminescence intensification with the help of a method often used for definition of the laser effect.

Let at first approximation the Doppler widening of spectral lines predominate over other widening mechanisms at low pressure. Width of a spectral line can be obtained according to the formula:

$$
\Delta v=\frac{2}{\lambda} \sqrt{\frac{2 \ln \mathrm{kT}}{\mathrm{m}}},
$$

where $\lambda$ is the length of a wave in meters, $m$ is the atom mass in kilograms, $\mathrm{T}$ is the Kelvin absolute temperature, $\mathrm{k}$ is the Boltzmann constant. Coefficient of radiation intensification $a(v)$ due to inversion can be computed by the formula:

$$
a(v)=\frac{1}{4} \lambda^{2} g_{k} a_{k i}\left[\frac{N_{k}}{g_{k}}-\frac{N_{i}}{g_{i}}\right],
$$

Where $g_{k}$ and $g_{i}$ are statistical weights of levels with populations $\mathrm{N}_{\mathrm{k}}$ and $\mathrm{N}_{\mathrm{i}}$, respectively. If the frequency corresponding to a given spectral line is $v_{0}$, spectral density $a_{k i}$, the first factor of the Einstein coefficient $A_{k i}$, then $\mathrm{a}_{\mathrm{ki}}$ is equal :

$$
a_{k i}=A_{k i}(\sqrt{\pi} \Delta v)^{-1} \exp \left[-\frac{\left(v-v_{0}\right)^{2}}{\Delta v^{2}}\right]
$$

The computed data for radiation intensification coefficient in $\mathrm{m}^{-1}$ units for the Fel spectral line center $\lambda=4072 \AA, \operatorname{MgI} \lambda=5184 \AA$, sodium D-lines, and CaII lines $\mathrm{H}$ and $\mathrm{K}$ are shown in Fig. 2 as a function of stellar magnitude.

Thus, for a A-meteor from Fig.1 from a stream of Leonids photographed by the author on November 17 , 1965 radiation intensification due to the above effect was about 150 per each kilometer. The numerical examples are shown in (Smirnov, 2000).

Hence, the meteor phenomena are accompanied by the following processes in different time scales:

1. Relatively slow processes of heat conductivity, mechanical rotation, splitting, and 
2. Considerably faster brightness fluctuations in the emerged clot of meteor radiating plasma, similar to gasdynamical laser radiation.

The flares caused by the first and second reasons differ both in nature and appearance depending on the character of radiation.

As was specified above, let's name "mechanical" flares type B and "laser" ones type A.

In Fig. 2 on the next page (Smirnov, 2007) the flares of $\mathrm{B}$ type are shown for rather slow fireballs when the plasma component of the meteor phenomenon does not show itself, as velocity is insufficient for its formation. The flares are formed due to mechanics of a meteor body rotation accompanied by fusion of its outside layers and discarding the fused substance into atmosphere. In Fig.1 (Smirnov, 2007) the A type flare for Leonids radiation with prevalence of a meteor plasma luminescence according to the Wien law with final flare is shown.

In (Abe et al., 2007) Fig. 1 features the bright Leonid's spectrum in which both types of flares are most likely inter-imposed. The topmost bright flare can be supposed to result from the A and B flares superimposition; a flare at the meteor path end and with the meteor complete disintegration corresponds to type A. In this way the figure shows the "laser" radiation factor at the meteor spectrogram.
Fig. 1 of this work shows all three meteor spectrograms discussed above. Comparison of spectrograms shows their correspondence to the above-said. Fig. 2 presents radiation intensification coefficients (on logarithmical scale) related to the meteor stellar magnitude (Smirnov, 2000).

\section{References}

Abe S., Ebizuka N., Yano H., Watanabe J., Borovicka J.: 2007, Adv. Space Res., 39, 4, 538-543.

Astapovich I.S. Meteor Phenomena in the Land Atmosphere. Fizmatgiz, Moscow, 1958.

Biberman L.M., Vorobjov V.S., Jakubov I.T. The Kinetics of Non-equilibrium Low-temperature plasma. Science. Moscow, 1982.

Chudnovsky A.F. Heat-physics of Soils. Science, Moscow, 1976.

Smirnov V.A.: 2007, Adv. Space Res., 39, 4, 526-532.

Smirnov V.A. The Spectra of Transient Atmospheric Luminous Phenomena: Meteors. Physico-Mathematical Literature, Moscow, 1994.

Smirnov V.A.: 2000, Proc. of IMC Frasso Sabino, International Meteor Organization, 59-71.

Smirnov V.A.: 2003, in: Proc. of IMC Frombork /eds. Olech A., Zloczewski K., Mularczyk K., Poland 26 - 29 September 2002. International Meteor Organization, 119 - 124 . 